\title{
Study on Definition of Sequence Limit in the Advanced Mathematics with Scaffolding Instructions
}

\author{
Ling Chen \\ College of Mobile Telecommunications Chongqing University of Posts and Telecom \\ China Chongqing 401520
}

Project introduction: Reform of higher education research project in Chongqing University of Posts and Telecommunications College of mobile（YTJG201627,YTJG201613,YTJG201626).

Keywords Scaffolding instruction; Teaching design; Limit of sequence

\begin{abstract}
With the needs of education reform, the traditional mathematics teaching model is no longer adapted to the development of the times, and scaffolding instruction is a new teaching mode arises at the historic moment. To define the limit in higher mathematics series as an example, studying the use of scaffolding instruction.
\end{abstract}

[CLC number] G642.4

[Document identifier code] A

\section{The Concept of Scaffolding Instruction}

Scaffolding instruction, also called support point teaching, is one of the most mature teaching methods under the constructivist mode. It is defined as: "scaffolding instruction should provide a conceptual framework for learners to construct knowledge understanding. The concept of this framework is needed to develop learners' further understanding of the problem. Therefore, it is necessary to decompose the complex learning tasks in advance so as to facilitate the gradual deepening of learners' understanding., ${ }^{,[1]}$

\section{Steps of Scaffolding Instruction Design}

Scaffolding instruction includes the following aspects

Scaffolding: around the current learning theme, in accordance with the requirements of the "nearest development zone" to establish a conceptual framework.

Feeling the situation: leading students into a certain problem situation (a node within the conceptual framework).

Independent exploration: let students explore independently. The exploration includes: determining various attributes related to a given concept, and arranging various attributes according to their importance.

Collaborative learning: group negotiation and discussion. On the basis of sharing the results of collective thinking, it can achieve a comprehensive and correct understanding of the current concepts, that is to complete the construction of the meaning of the knowledge.

Evaluation: the evaluation of learning include learning evaluation of students' self evaluation and learning group of individuals, the evaluation content includes: autonomous learning ability; the team makes contributions to the completion of learning; knowledge of meaning construction. ${ }^{[1]}$

\section{Instructional Design of Scaffolding Instruction}

The use of "proximal development zone" to the creation of a cognitive imbalance, to stimulate interest in learning

The development of students' cognition is the process of the balance of ideas being constantly destroyed and constantly reaching a new equilibrium state. In the process of teaching, teachers should give new situations in time to break the original cognition of students, and make students acquire the motivation and interest to get new knowledge. 


\section{Cultivating students' ability of migration according to the actual "scaffolding"}

Background bracket - giving the migration object

New knowledge is always in the students' existing knowledge, therefore, teachers can use common sense support design background in teaching to let students understand the old knowledge transfer to new knowledge needing to achieve cognition. And it can cite many similar examples to understand the urgency of the need for new knowledge.

Problem scaffold - a sense of transfer

Problem scaffold is the most frequently used scaffold. It has a complete structure and is able to pay attention to every detail. In the original knowledge and experience as the background, the students learn new knowledge when teachers expect students in the old knowledge to new knowledge in the migration process may encounter difficulties, is given in the background as the basis from different levels and different angles to set up problems with support without broken from the old students that will be solved knowledge gradually migrated to the new knowledge to understand and master.

Image support - stimulus migration

Image is a very intuitive form of expression, especially for the course of mathematics which often use tables, images, Wayne diagrams, mathematical models and so on. In the process of teaching, teachers use the intuitive way to express all kinds of information in teaching encountered, it can make the students thinking more rigorous and can be used for the interpretation of knowledge, analysis and summary. In a certain context, through the students themselves, and actively build the link between the old and new knowledge to stimulate students to knowledge transfer occurs.

Tool support - developing the habit of transfer

The tool holder is refers to the provided by teacher, such as dialogue, exchange, exhibition, PPT show, animation, software programming, electronic whiteboard, reference books, cyber source and props models, these tools can give students knowledge assimilation and adaptation, internalization and construct the concept, and it can make many abstract concepts and theorems of mathematics curriculum in specific, geometry and visualization making students form the habit of migrating.

Building scaffold - improving migration ability

The new capacity is built based on previous acquisition ability, in the teaching, it should be as far as possible to improve the construction of new knowledge, and teachers should strive to create support to let students actively participate in learning activities, but also can use the reverse thinking, the knowledge transfer to other corresponding issues making the learning content that can be built into the more extensive knowledge.

Inquiry learning: Students' thinking ability can be improved in the bracket under the action of bracket

Independent exploration - learn to think independently

Learning in the University can not always rely on the help of the teacher. On the simple question, students should explore and study independently, and sum up the conclusion as much as possible. When students feel independent exploration in times of trouble, teachers can build support timely to provide students with some help in the exploration process, at the same time removed at the appropriate time frame to let the students to explore independently and improve the ability of independent thinking.

Collaborative learning - sharing collective wisdom

Some knowledge for students to think independently is very difficult, so it can let students learn from each other using collective power to cooperate and exchange to explore new knowledge and conclusion and share the wisdom of the collective.

\section{The Case of Scaffolding Instruction Model - The Limit of Sequence}

The limit is the research method of higher mathematics, and the limit of the sequence is the basis of the limit function. The application of the sequence limit as an example to explain in detail the scaffolding instruction mode. 
Bracket 1(3.1):Learn to find the first sum of a sequence $n$, for example first ten terms $\left\{\frac{1}{2^{n}}\right\}$ and $\frac{1}{2}+\frac{1}{2^{2}}+\cdots+\frac{1}{2^{10}}=1-\frac{1}{2^{10}}$, then the sum of infinite numbers of this sequence is equal $\frac{1}{2}+\frac{1}{2^{2}}+\cdots+\frac{1}{2^{n}}+\cdots=$ ?

Note: most of the students will answer that it is infinite, so the creation of unbalanced cognition.

Primary school has been aware of the perimeter of a circle is $2 \pi R$, why?

Bracket 2(3.2 (1)):Cyclotomy "cut in thin lose one, then again, so that can not be cut, and the circumference fit without lose"; "a husband cut foot of the whip, on the section of the half, and there will be no end".

Bracket 3 (3.2 (2)): The method of finding the circumference of a circle is given by cutting circle. The relation between a number and the sum of infinitely many numbers is given. How to do it?

Bracket 4 (3.3 (2)):The student groups are in collaborative discussion, and during the discussion, teachers appropriate to give new support.

Bracket 5 (3.2 (3)):For cutting circle method, when the students discussed for some time but has no target, give the image bracket (Figure 1), How can the perimeter of the inscribed regular polygon $C_{n}$ change into the perimeter of the circle C? And then give the image bracket after a certain period of time (Figure 2).

Note: "when the number of edges of inscribed regular polygon is infinitely increased, its circumference is infinitely close to the circumference of the circle $C_{n} \stackrel{n \rightarrow \infty}{\rightarrow} C$ "

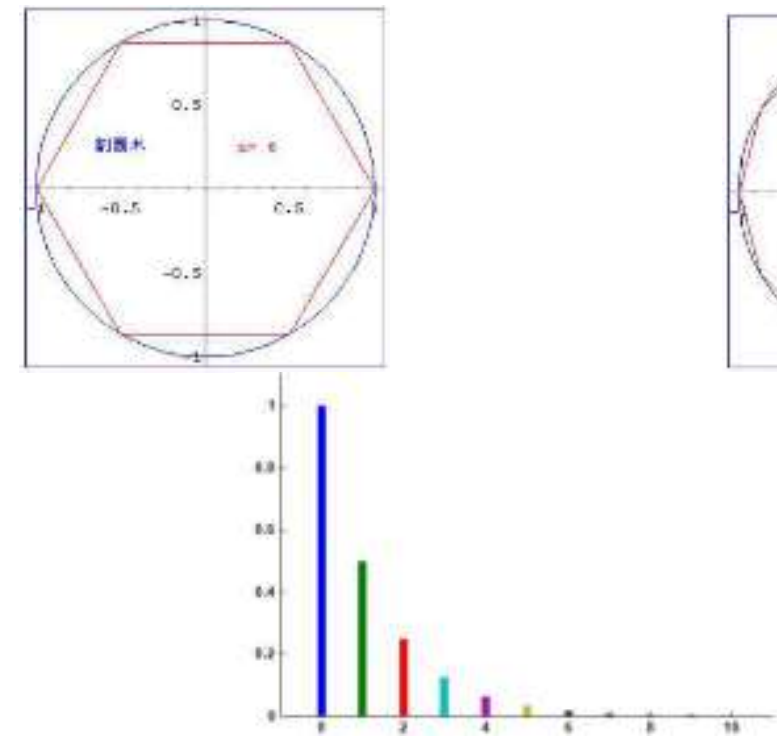

Figure 1.
Figure 2.

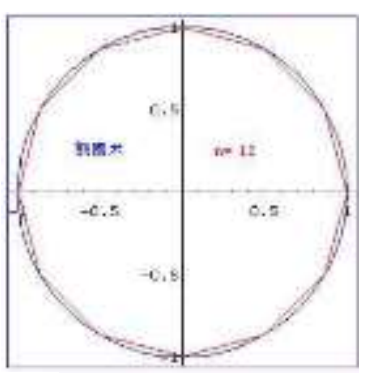

Figure 3.

Bracket 6 (3.2 (4)):For interception problem, when students discuss a certain period of time but still no way of thinking with a strip of paper demonstration, each half, after several times getting the results (Figure 3).

Note: the conclusion is that when the time is infinite, the length of the remaining foot is infinitely close to 0 , which means $\frac{1}{2^{n}} \stackrel{n \rightarrow \infty}{\rightarrow} 0$.

Bracket 7 (3.2 (3)):Depicting figure of sequence in $\left\{\frac{1}{2^{n}}\right\}$ Cartesian coordinate system? Students think, and teachers guide (Figure 4). 


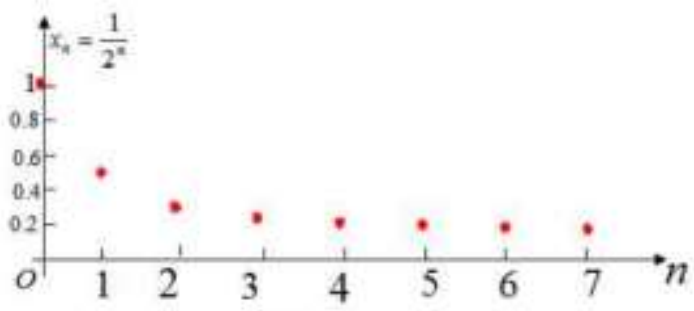

Figure 4 .

Table 1

\begin{tabular}{|c|c|c|c|}
\hline \multicolumn{4}{|c|}{$x_{n}=1-\frac{1}{n}, \frac{1}{n}=\left|1-\frac{1}{n}-1\right|=\left|x_{n}-1\right|$} \\
\hline$\varepsilon>\left|x_{n}-1\right|$ & & $\frac{1}{1000}>\left|x_{n}-1\right|$ & \\
\hline$n>N$ & $n>100$ & & $n>10000$ \\
\hline$\varepsilon$ & $\frac{1}{100}$ & $\frac{1}{1000}$ & $\frac{1}{10000}$ \\
\hline$\forall_{-}, \exists_{-}$, when $n>_{-}, \quad$ then $\left|x_{n}-1\right|<_{-}$. \\
\hline
\end{tabular}

Bracket 7 (3.2 (5),3.3): When all sequences $\left\{x_{n}\right\}$, and $n \rightarrow \infty$, are they infinitely close to a constant? With group cooperation, drawing the image of a sequence of numbers $\left\{1-\frac{1}{n}\right\},\left\{2^{n}\right\},\left\{(-1)^{n}\right\}$ in the Cartesian coordinate system? And observing when $n \rightarrow \infty$, the variation characteristics of general items of a sequence.

Note: draw a conclusion "when $n \rightarrow \infty$, some general infinite series is close to a constant, some series of general infinitely close to infinity, and some sequences tend to be indefinite."

Bracket 8 (3.3): Let the students fill in Table 1, then cooperate to fill between students, and the teacher guides everyone to perfect together to draw conclusions.

Note: the definition of limit of sequence $\left\{1-\frac{1}{n}\right\}$ is summed up, $\forall \varepsilon>0, \exists N>0$, when $n>N$, then $\left|\left(1-\frac{1}{n}\right)-1\right|<\varepsilon$ and it is noted as $\lim _{n \rightarrow \infty}\left(1-\frac{1}{n}\right)=1$.

Bracket 9 (3.2 (5)):Through the special, summing up the definition of sequence limit under normal conditions, $\forall \varepsilon>0, \exists N>0$, when $n>N$, then $\left|x_{n}-A\right|<\varepsilon$, and it is noted as $\lim _{n \rightarrow \infty} x_{n}=A$.

\section{Conclusion}

There is a method of teaching and no way to teach. Lesson preparation is not only the accumulation of simple knowledge, but also need to have a certain understanding of students, and lesson preparation is not a simple back teaching materials, the key is to recite students. Establish a suitable scaffolding for their class students, at the appropriate time to dismantle scaffolding and establish new scaffolding, but also several scaffolding can be simultaneous. Through scaffolding and other teaching methods, teachers cultivate students' ability of active learning, active thinking, independent analysis, judgment and summary in order to meet the needs of the new society.

Note: "3.2 (1)" means the theory of bracket comes from 3.2 "1) background bracket - giving the migration object". 


\section{Reference}

[1] (US) Wuhrer Falk, He Xianyou et al. Educational psychology[M].China Light Industry Press:2014.

[2] Department of mathematics, Tongji University. Higher mathematics book (Sixth Edition)[M].Tongii University Press:2010.

[3] Griffiths, David; Owen, Martin. Environment challengs: making a difference in the classroom[D].DGXIII Proceedings of the computer Assisted Learning(CAL97) Conference,1997,(3):1-8.

[4] Xueshan Li. Teaching strategies based on Constructivism Theory-Taking the course of Advanced Mathematics as an example[J].Journal of Southwestern Normal University(natural science edition),2014.2,39(2):154-157. 